\section{Economics of Cancer Care}

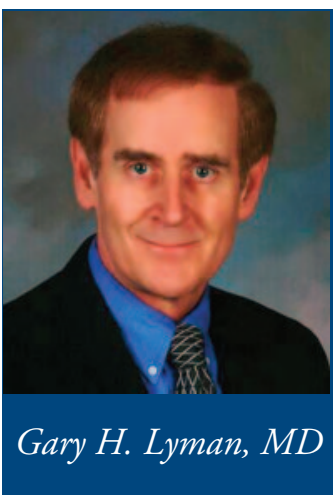

By Gary H. Lyman, MD

The impact of cancer and cancer treatment on health care costs has increasingly gained the attention of providers and payers as well as patients and their families. Health care costs include the direct costs of receiving medical care and the nonmedical costs incurred while receiving care such as transportation or childcare expenses. In addition, there are indirect costs associated with the morbidity of cancer care such as days lost from work for the patient or caregiver. Intangible costs such as pain and suffering and loss of companionship are difficult to measure but very real to the patient and family.

Economic studies are most useful when an intervention is associated with a better clinical outcome but at greater cost or when it is associated with a lower cost but the same or even less favorable outcome. ${ }^{1}$ When clinical effectiveness is the same, a cost-minimization analysis may be utilized to define the approach with the lowest cost. Where clinical or qualityadjusted effectiveness differ, economic analyses are generally based on cost-effectiveness, which estimates the added cost per life year gained. ${ }^{1}$ Such approaches attempt to compare and identify the least costly approach with the greatest effectiveness.

To fully understand discussions of costs and costeffectiveness, one must know the perspective from which the study was conducted from that of society, the payer, the institution, the provider or the patient and family. Where possible, economic analyses associated with controlled clinical trials should be conducted before wide dissemination of new technologies especially when the potential resource utilization and costs are large. ${ }^{2,3}$ The care taken in the design and analysis of clinical trials provide the best available information on resource utilization and treatment efficacy.

Health care costs have risen dramatically over several decades, now approaching $\$ 2$ trillion annually with the costs of cancer care representing a approximately $10 \%$ or roughly $\$ 200$ billion. Cancer care costs are greatest during the period of initial treatment immediately following diagnosis and during the last few months before death. The costs associated with cancer treatment must consider both the costs of treatment but also the cost of managing disease progression or recurrence as well as the cost of treatment-related toxicity. 4,5 Unfortunately, the economic value of nonmedical costs such as time required to obtain care or indirect and out-of-pocket expenses or loss of work productivity, are rarely considered.

To address the situation described in a recent article, ${ }^{6}$ colorectal cancer accounts for approximately $10 \%$ of invasive cancers as well as cancer-related deaths in adults. Until the 1990s, fluorouracil was the mainstay of chemotherapy for colorectal cancer. However, the costs of colorectal cancer treatment have increased dramatically over the past decade in large part due to the dissemination of several new expensive agents including irinotecan; oxaliplatin; capecitabine; and two monoclonal antibodies, bevacizumab and cetuximab. Schrag has pointed out that the average wholesale price for these new agents over an 8-week course may range from $\$ 10,000$ to $\$ 30,000$. $^{7}$ Despite the concerns of many about how and who will pay for such escalating costs, Yabroff et al reported that the problem is even worse from the economic perspective of the patient, family, and society. ${ }^{8}$ FOLFOX (fluorouracil, leucovorin, oxaliplatin) is rapidly becoming standard of care for stage III colon cancer and involves a 6-hour treatment time and a 46-hour home infusion generally every 14 days. ${ }^{9}$ In fact, hospitalization represents approximately two-thirds of the estimated time costs. The nonmedical costs were found to approach as much as half the direct medical costs during the terminal phase of illness with hospitalization representing the single largest contribution.

Costs should never become the primary focus of physicianpatient discussion and should not alter recommendations for proven effective treatment in an effort to ration care. Efficacy and safety rather than costs remain the major focus of US Food and Drug Administration review and approval of new oncologics and costs are mentioned only for information purposes in clinical practice guidelines from ASCO. ${ }^{10}$ Rather, we must look for strategies that may limit the economic impact of these emerging technologies. Industry should be encouraged to reassess pricing policies while relief should be sought from prohibitions on negotiating prices and importation of known safe drugs. In addition, investigators should be encouraged to search for improved prognostic and predictive molecular and genetic markers to complement conventional clinical indicators. ${ }^{11}$ Such strategies have the potential of improving both the effectiveness and cost effectiveness of valuable treatments by targeting agents toward patients most likely to benefit from specific treatments. ${ }^{12}$ However, the challenge for providers in communicating both the complex clinical situations and treatment options but also the ensuing costs of care in a useful and caring manner will remain and perhaps become an even greater challenge for the next generation of oncologists. 


\section{References}

1. Lyman GH, Djulbegovic B: Understanding economic analyses. EvidenceBased Oncol 2:2-5, 2001

2. Lyman GH: Methodological issues related to health economic analysis in controlled clinical trials, in Crowley J (ed): Handbook of Statistics in Clinical Oncology Marcel Dekker, 2001 pp 291-320

3. Lyman GH: Economic analysis of randomized controlled trials. Curr Oncol Rep 3:396-403, 2001

4. Lyman GH, Berndt E, Crown W, et al: The economic burden of anemia in cancer patients receiving chemotherapy: Value in health 8:149-156, 2005

5. Kuderer NM, Dale D, Crawford J, et al: The morbidity, mortality and cost of febrile neutropenia in cancer patients. Cancer 106:2258-2266, 2006

6. Talking with patients about the cost of cancer care. J Oncol Pract 3:122-123, 2007
7. Schrag D: The price tag on progress: Chemotherapy for colorectal cancer. N Engl J Med 351:317-319, 2004

8. Yabroff KR, Warren JL, Knopf K, et al: Estimating patient time costs associated with colorectal cancer care. Med Care 43:640-648, 2005

9. Lyman GH: Time is money for both the healthy and the sick. Medical Care 43:637-639, 2005

10. ASCO Health Services Committee Clinical Practice Guideline Handbook. Alexandria, VA, American Society of Clinical Oncology

11. Lyman GH, Kuderer NM: Gene Expression Profile Assays as Predictors of Recurrence Free Survival in Early Stage Breast Cancer: A Meta-Analysis. Clin Breast Cancer 7:372-379, 2006

12. Lyman GH, Cosler LE, Kuderer NM, et al: Impact of a 21-gene RT-PCR assay on treatment decisions in early-stage breast cancer: An economic analysis based on prognostic and predictive validation studies. Cancer 109:1011-1018, 2007

\section{JOP Online Moves to HighWire Press Platform}

Oncology professionals benefit from a more complete, useful, and effective online browsing and searching experience since JOP Online has moved to the HighWire Press platform.

The new Web site http://jop.ascopubs.org will continue to house JOP articles back to Volume 1, Issue 1 (May 2005), but will now allow readers to take advantage of robust online functionality:

- The ability to search the JOP archive by citation, author, topic, issue, keyword, or DOI

- The option to simultaneously search across JOP, JCO, and more than 1,000 other top medical journals hosted by HighWire Press using a single login

- The power to customize reading and research by utilizing optional e-mail alerts, PDA downloads of JOP content, and PowerPoint downloads of figures 